\title{
NEXT NEAREST COUPLING FROM ANALYTICAL EXPRESSIONS FOR THE APT LINAC ${ }^{*}$
}

\author{
Paul Greninger ${ }^{\#}$ \\ General Atomics, San Diego, CA
}

\begin{abstract}
In order to design any accelerator one should know three constants, $\Delta \mathrm{f}, \mathrm{k}$ and $\mathrm{kk}$. The $\Delta \mathrm{f}$ determines the noslot cavity frequency i.e., the cavity frequency will drop with the introduction of coupling slots. Constant $\mathrm{k}$ determines the coupling between on-axis cavity to cavity. Constant kk determines the next nearest coupling from onaxis cavity to cavity. In a Couple Cavity Drift Tube Linac the quantity $\mathrm{kk}$ is minuscule. However in a Coupled Cavity Linac [1] because of the close proximity of the coupling cavity slots the quantity kk or next nearest becomes significant. Recent work at Los Alamos National Laboratory has employed a perturbation technique by J. Gao [2]. Good values of $\mathrm{k}$ are obtained from analytical expressions. With less success the quantity $\Delta \mathrm{f}$ can be calculated. It is the purpose of this paper to extend this type of analysis to include $\mathrm{kk}$ in a CCL. The approach will be to calculate the dipole induced in the slot by the field in the accelerating cavities. Next calculate an interaction energy between the two dipoles and finally employ Slater perturbation [3]. The calculated value of $\mathrm{kk}$ is approximately 0.002 , a reasonable number compared to experimental data from the LAMPF [4] accelerator at LANL.
\end{abstract}

\section{INTRODUCTION}

The motivation for the present paper is to calculate the frequency of coupled cavity structures, rather than measure them. Presently, there is work being performed at Los Alamos National Laboratory in the design of CCDTL [1] for the Accelerator Production of Tritium project. Good values of nearest coupling are obtained by a perturbation technique [2], which involves fields from Superfish models of the on-axis cavity, and the coupling cavity. These fields are entered into analytical expressions, which yield the coupling constant, and the $\Delta f$, due to slots. The Superfish cavity frequency is tuned beyond the nominal frequency such that when the slots are introduced the end result is the coupled cavity structure that will resonate at $700 \mathrm{MHz}$. The unperturbed cavity dimensions, along with the slots are iterated until a selfconsistent solution exists at the nominal cavity

* supported by D.O.E. under the A.P.T. program.

"Email: grenin@gat.com frequency. It is the goal of the present paper to extend this work to the design of the CCL, where next nearest coupling is significant, and affects the mode spectrum. The next nearest neighbor coupling constant presented here is based upon a theoretical calculation. Cold models are presently being built for experimental verification. Next nearest neighbor coupling can then be estimated from the mode frequencies and program DISPER [5].

\section{APPROACH TO COUPLING}

J. Gao has published a paper calculating nearest neighbor coupling from analytical expressions [2]. Onaxis cavity coupling is calculated from Superfish models of the on-axis cavity and the coupling cavity. The fields in these models set up a pair of interacting electric and magnetic dipoles in the coupling slots. The energy of a self-induced dipole of one cavity interacting with the fields from another cavity is related to the energy term in the Slater perturbation formula. Analytical expressions for the dipole moments set up in elliptical slots came from an earlier paper by Hans Bethe [6]. We will extend the present technique to calculate next nearest neighbor coupling.

\section{FORMULAS}

Because the slots are only in a region of high magnetic flux, we concern ourselves solely with the magnetic dipole term. We shall calculate the coupling from one on-axis cavity to another on-axis cavity. Some coupling may occur through the drift tubes. This can be calculated with Superfish and we found this term negligible.

The magnetic field intensity of a dipole can be expressed as [7]

$$
H=\frac{1}{4 \pi} \frac{3 n(n \cdot m)-m}{|x|^{3}}
$$

(m.k.s.) (1)

Where $\mathrm{m}$ is the magnetic dipole moment, and $\mathrm{n}$ is the unit vector in the $\mathrm{x}$ direction. The interaction energy between dipoles can be derived by summing the effect of magnetic dipole 1 in the field of 2 , and conversely dipole 2 in the field of 1 . The one-half coefficient in front of the parenthesis in (2) for time averaging. 


$$
\Delta W=\frac{1}{2}\left(\frac{\mu_{0}}{2} m_{1} \cdot H_{2}+\frac{\mu_{0}}{2} m_{2} \cdot H_{1}\right)
$$

Employing the notation,

$$
\Delta W=\Delta W_{1,2}+\Delta W_{2,1}
$$

the quantity $\Delta \mathrm{W}_{1,2}$ is formed by substituting the value of dipole 1 into the $\mathrm{H}$ field of 2 .

$$
\Delta W_{1,2}=\frac{\mu_{0}}{16 \pi} \frac{m_{1} \cdot\left(3 n\left(n \cdot m_{2}\right)-m_{2}\right)}{|x|^{3}} .
$$

Performing the vector algebra and expressing the total energy

$$
\Delta W=\frac{\mu_{0}}{16 \pi}\left(\frac{3\left(n \cdot m_{1}\right)\left(n \cdot m_{2}\right)-m_{1} \cdot m_{2}}{|x|^{3}}\right)+\Delta W_{2,1}
$$

The $\mathrm{H}$ field and the induced dipole orientation for the $\pi / 2$ mode are shown in Fig. 1. Our notation will be $A C=$ Accelerating Cavity, $C C$ $=$ Coupling Cavity.

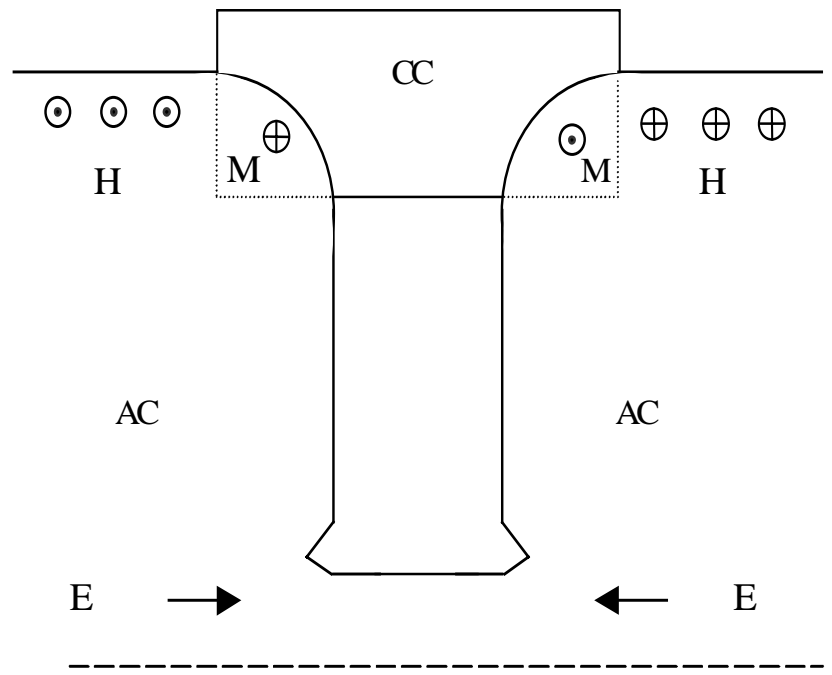

Figure 1: Diagram for Two Coupled Cavities in the $\pi / 2$ Mode. listed as:

From the J. Gao paper the value of $m$ is

$$
m_{1}=\frac{\pi\left(\frac{l_{1}}{2}\right)^{3} e_{0}^{2}}{3\left(K\left(e_{0}\right)+E\left(e_{0}\right)\right)} H_{1}
$$

Where $e_{0}^{2}=1-(l / w)^{2}, I$ is the length of the slot, $w$ is the width of the slot, $K\left(e_{0}\right)$ and $E\left(e_{0}\right)$ are elliptical integrals, and $H_{1}$ is the unperturbed field in the AC. A diagram of a slot is listed below.

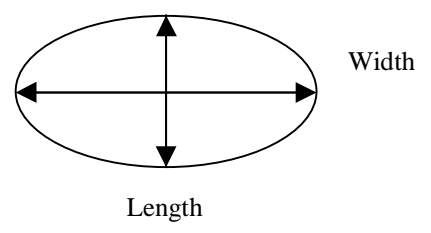

Figure 2. Diagram for Slot Geometry.

The unit normal, $n$, from one slot to another is perpendicular to the $\mathrm{H}$ in the slots, therefore the quantity $n \cdot m$ vanishes.

To calculate $\mathrm{kk}$ look at the mode separation of two AC cavities in the 0 and the $\pi$ mode. Assume next nearest coupling only. Then in Fig. 1 without the presence of the coupling cavity, the two on-axis cavities behave as if they are in the $\pi$ mode. From Slater Perturbation the frequency of a perturbed mode can be written as

$$
\omega_{\text {mode }}^{2}=\omega^{2}\left(1+\frac{2}{U} \Delta W\right)
$$

For the $\pi$ mode the m's are anti-parallel therefore

$$
\omega_{\pi}^{2}=\omega^{2}\left(1+\frac{\mu_{0}}{4 \pi} \frac{m_{1} \cdot m_{2}}{U|x|^{3}}\right)
$$

For the zero mode, the m's have the same direction.

$$
\omega_{0}^{2}=\omega^{2}\left(1-\frac{\mu_{0}}{4 \pi} \frac{m_{1} \cdot m_{2}}{U|x|^{3}}\right)
$$




$$
2 \omega \Delta \omega=\omega_{\pi}^{2}-\omega_{0}^{2},
$$

substituting,

$$
\Delta \omega=\omega \frac{\mu_{0}}{4 \pi} \frac{m_{1} \cdot m_{2}}{U|x|^{3}}
$$

Finally, with

$$
\begin{aligned}
& k k=\frac{\Delta \omega}{\omega}, \\
& k k=\frac{\mu_{0}}{4 \pi}\left(\frac{\pi\left(\frac{l}{2}\right)^{3} e_{0}^{2}}{3\left(K\left(e_{0}\right)+E\left(e_{0}\right)\right)}\right)^{2} \frac{H_{a c}^{2}}{|x|^{3} U_{a c}} .
\end{aligned}
$$

In analogous fashion, a $\mathrm{CC}$ to $\mathrm{CC}$ term can also be calculated. This term tends to be less because the coupling cavities are usually staggered off the axis of symmetry, and their slots further apart.

If more cells are added a bi-periodic structure can be formed. The addition of more cells distances us from half-cell end boundary conditions. These end cells do not have the proper boundary conditions to fully reveal next nearest coupling. With all on-axis cavities tuned to one frequency $\omega_{1}$, and all coupling cells tuned to another frequency $\omega_{2}$, the $\pi / 2$ modes are related by the formula [8]:

$$
\begin{aligned}
& \omega_{\pi / 2}^{2}=\frac{\omega_{1}^{2}}{1-k k_{a c}} \\
& \omega_{0}^{2}=\frac{\omega_{2}^{2}}{1-k k_{c c}}
\end{aligned}
$$

The difference between the two modes is the stop band. In a well tuned acclerator the $\pi / 2$ mode for the CC cells is not present because there is no stored energy in them.

\section{RESULTS}

Presently we are calculating a value of 0.00188 for an on-axis cavity with $\beta=.42277$ in our CCL. This number is in line with the final tuning data for the Los Alamos LAMPF accelerator [4] where for $\mathrm{k}=.05, \mathrm{kk}$ is in the range 0.001 to 0.005 .

\section{CONCLUSION}

Reasonable values of the next nearest neighbor coupling coefficient can be calculated approximating the slots as magnetic dipoles and applying the Gao theory. Our value of next nearest neighbor coupling falls within the range 0.001 to 0.005 for values stated in the literature for a CCL with $5 \%$ coupling.

\section{REFERENCES}

References:

[1] Thomas Wangler, RF Linear Accelerators. New York: Wiley, 1998, pg. 103

[2] J. Gao, "Analytical Formulas for the Resonant Frequency Changes due to Opening Apertures on the Cavity Walls," Nuclear Instruments \& Methods in Physics Research. A311 (1992) 437-443

[3] John C. Slater, Microwave Electronics. New York: Van Nostrand, p. 81

[4] LAMPF 805-MHz Accelerator Structure Tuning and its Relation to Fabrication and Installation.

LA- 7915-MS informal report.

[5] Computer Program "Disper", written by James Billen, of Los Alamos National Laboratory.

[6] H.A. Bethe, Phys. Rev 66 (1944) 163.

[7] J. D. Jackson, Classical Electrodynamics, New York: Wiley, $1^{\text {st }}$ Ed., p. 147

[8] D.E. Nagle, E.A. Knapp, and B. C. Knapp, "Coupled Resonators Model for Standing Wave Accelerator Tanks," Review of Scientific Instruments, Vol. 38, Number 11, Nov. 1967 